\title{
EXPERIMENTAL STUDIES OF THE ELECTRICAL CONDUCTIVITY AND PHASE TRANSITION IN $\mathrm{Fe}_{3} \mathrm{O}_{4}^{*}$
}

\author{
B. J. Evans ${ }^{\dagger}$ \\ Department of Geology and Mineralogy \\ University of Michigan \\ Ann Arbor, Michigan 48104
}

\section{ABSTRACT}

Above the Verwey transition the conduction electrons in $\mathrm{Fe}_{3} \mathrm{O}_{4}$ are described best by a band model. Those experimental data interpreted as indicating localized hopping conduction are either not susceptible to definitive interpretations or have been incorrectly interpreted. A band model is also appropriate above the Neel temperature and the influence of magnetic order on the conduction mechanism is only of minor significance. The Verwey transition is complex and involves both electronic and structural aspects. The temperatures of these transitions may be different or identical depending upon the purity of the $\mathrm{Fe}_{3} \mathrm{O}_{4}$. Good correlations exist between Mössbauer effect and thermal properties measurements concerning the complexity and qualitative characteristics of the Verwey transition. Resistivity measurements appear to confirm the Mössbauer effect and thermal properties measurements.

\section{INTRODUCTION}

There have been several timely reviews ${ }^{1-3}$ of electrical conduction mechanisms in transition metal oxides, including $\mathrm{Fe}_{3} \mathrm{O}_{4}$, whose electrical conductivities undergo dramatic changes in a small temperature interval. And while one review has been devoted exclusively to $\mathrm{Fe}_{3} \mathrm{O}_{4}{ }^{4}$, a comprehensive and critical appraisal of most of the available electrical, magnetic and thermal properties data of $\mathrm{Fe}_{3} \mathrm{O}_{4}$ has been lacking. Nonetheless, the previous reviews ${ }^{1-3}$ do provide an adequate appraisal of the electrical properties measurements that were available as of 4 to 6 years ago. Since these earlier reviews, however, there has been a great increase in investigations, both experimentally and theoretically, of $\mathrm{Fe}_{3} \mathrm{O}_{4}$; and consequently, considerably more facts and different ideas have been generated regarding the electrical properties and low temperature polymorphism in $\mathrm{Fe}_{3} \mathrm{O}_{4}$. These new results will be the primary concern of this inquiry. Even though these new data when simply juxtaposed are rather confusing and contradictory, it will be shown in the following that a reasonably consistent picture as to the electrical conduction mechanism and the low temperature polymorphism emerges from a critical analysis of these data, especially when compared with the results of some very recent studies.

It is encouraging that most of the data resulting from earlier studies of the electrical and magnetic properties of $\mathrm{Fe}_{3} \mathrm{O}_{4}$ above and in the region of the Verwey transition $5,6, T_{V}$, have been confirmed by recent investigations ${ }^{7,8}$. Therefore, our consideration of data obtained in the temperature interval $T_{\mathrm{V}}<T<T_{N},\left(T_{N}\right.$ = Neel temperature) will be directed primarily at resolving several alternative interpretations of the conduction mechanism rather than a detailed consideration of the data thermselves. Ideas concerning the structure of the low temperature phase ${ }^{9}$, below $T_{\mathrm{V}}$, have, however, been substantially altered by more recent studies 10 . Thermal property measurements in the region of the Verwey transition have also indicated a complexity beyond that previously reported ${ }^{11}$. The second major objective of this paper is to establish that the low temperature phase transition is a complex one. In this instance, data only recently available must be considered; these data result from different property measurements on identical samples and the complexity of the phenomena appears to be firmly established.

The principal conclusions of this investigation are the following. For the temperature interval
$\mathrm{T}_{\mathrm{V}}<\mathrm{T}<\mathrm{T}_{\mathrm{H}}$, a band description is appropriate for the conduction mechanism in $\mathrm{Fe}_{3} \mathrm{O}_{4}$. Previous data cited as indicating a hopping mechanism are found to be either incorrectly interpreted or to have resulted from poor samples. The conclusion reached for the temperature interval $\mathrm{T}_{\mathrm{V}}<\mathrm{T}<\mathrm{T}_{\mathrm{N}}$ receives additional support from Mössbauer and resistivity measurements for $T>T_{F}$. For $T<T_{V}$, a band description of the conduction mechanism is also supported by the available data, but this conclusion is less definitive than that reached for $\mathrm{T}_{\mathrm{V}}<\mathrm{T}<\mathrm{T}_{\mathrm{N}}$. In the region of the verwey transition, there appear to be two phase transitional phenomena: one related in the limiting case to purely electronic phenomena and another related to purely structural (crystal symmetry, site symmetry and atomic positional coordinates) phenomena. The phase transitional phenomena associated with the electronic state of the conduction electrons are more sensitive to the presence of impurities and defects than the structural transition. As expected for a structural transition in which there is a substantial lowering of the symmetry of the structure and changes in the atomic positional coordinates of most, if not all, of the ions, impurities on either the A or B sites have significant influences on temperatures and energy changes associated with the transition.

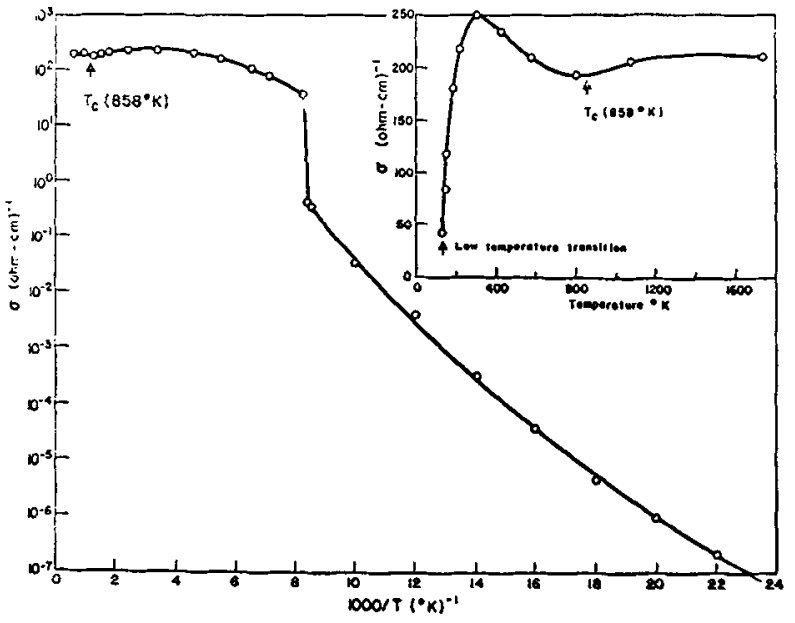

Fig. 1. Temperature dependence of the DC conductivity of single-crystal magnetite (ref. 6).

$$
\text { TEMPERATURE INTERVAI } \mathrm{T}_{V}<\mathrm{T}<\mathrm{T}_{\mathrm{N}}
$$

As might be expected, most of the available data of any sort has been obtained in this temperature interval ${ }^{6}$. The most important result in this region is the temperature dependence of the resistivity ${ }^{6}$ shown in Fig. 1. Recent investigations have confirmed the early measurements ${ }^{7}$, and provided more precise Hall effect and thermopower data ${ }^{12}$. The central question regarding the electrical conductivity in this region has been whether to describe it in terms of a localized hopping model or in terms of a band model. Until relatively recently, the description of the conduction mechanism in terms of a hopping model was rather qualitative and appeared to be based almost wholly on the conductivity being activated, apparently, and on the early suggestion 5 that the high electrical conductivity resulted from rapid electron interchange between the $\mathrm{B}$ site $\mathrm{Fe}^{2+}$ and $\mathrm{Fe}^{3+}$ ions. Fe $\mathrm{F}^{57}$ nuclear gamma-ray resonance 13 measurements soon after the discovery of the Mossbauer effect demonstrated the absence of distinct $\mathrm{Fe}^{2+}$ and $\mathrm{Fe}^{3+}$ ions on the octra- 
hedral site in $\mathrm{Fe}_{3} \mathrm{O}_{4}$. This result was interpreted in terms of a hopping model but is equally consistent with a band model in which there is significant screening of the ion cores. That the isomer shift and hyperfine field of the ${ }^{57} \mathrm{Fe} \mathrm{B}$ site pattern are intermediate to those of an isolated $\mathrm{Fe}^{3+}$ and $\mathrm{Fe}^{2+}$ ion of fers no unique support of a hopping model. Similar hyperfine fields and isomer shifts can be produced by appropriate screening. In addition, the hyperfine field and isomer shift of a hypothetical $\mathrm{Fe}^{2+}$ ion in $\mathrm{Fe}_{3} \mathrm{O}_{4}$ are of rather uncertain magnitudes and the magnetic hyperfine splitting of what may be considered an $\mathrm{Fe}^{2+}$ ion below $\mathrm{TV}_{\mathrm{V}}{ }^{4}, 16$ corresponds to a magnetic hyperfine field somewhat larger than that observed in other spinels that contain $\mathrm{Fe}^{2+}$ ions ${ }^{17}$.

The crucial experiment in establishing support for a hopping model was the measurement of the temperature dependence of the linewidth of the ${ }^{57} \mathrm{Fe} B$ site Móssbauer spectrum ${ }^{18}$. It has been confirmed in almost every Mössbauer study of $\mathrm{Fe}_{3} \mathrm{O}_{4}$, that the $\mathrm{B}$ site pattern has larger linewidths, $\Gamma_{B}$, than those of the A site pattern, $\mathrm{r}_{\mathrm{A}}$. On the assumption that there was no broadening of the A site linewidth, some function of the difference between $\Gamma_{A}$ and $\Gamma_{B}$, given by Eq. 1 , could be taken as measure of the $\mathrm{Fe}^{3+}(\mathrm{B})-\mathrm{Fe}^{2+}(\mathrm{B})$ electron hopping relaxation time

$$
\Delta \Gamma=\tau_{s} \Delta^{2} / 2
$$

where $\Delta \Gamma=\Gamma_{-}-\mathrm{I} ; \Delta$ is the difference in the assumed frequencies of the unrelaxed $\mathrm{Fe}^{2+}$ and $\mathrm{Fe}^{3+}$ lines associated with $\Gamma_{B}$ of the relaxed line and $\tau$ is the electron hopping relaxation time. Questionable assumptions regarding the hyperfine field isomer shift of an hypothetical $\mathrm{Fe}^{2+}$ ion in $\mathrm{Fe}_{3} \mathrm{O}_{4}$ figured prominently in this interpretation of the data. At any rate, the quantity ( $\left.\Gamma_{B}^{2}-\Gamma_{A}^{2}\right)$ increased with decreasing temperature and at $298 \mathrm{~K}$ a hopping time of about 1 nanosecond was deduced ${ }^{18}$. The conductivity calculated on the basis of this measurement of the relaxation time for the hopping mechanism was found to be two orders of magnitude less than the experimentally measured conductivity. This first linewidth study

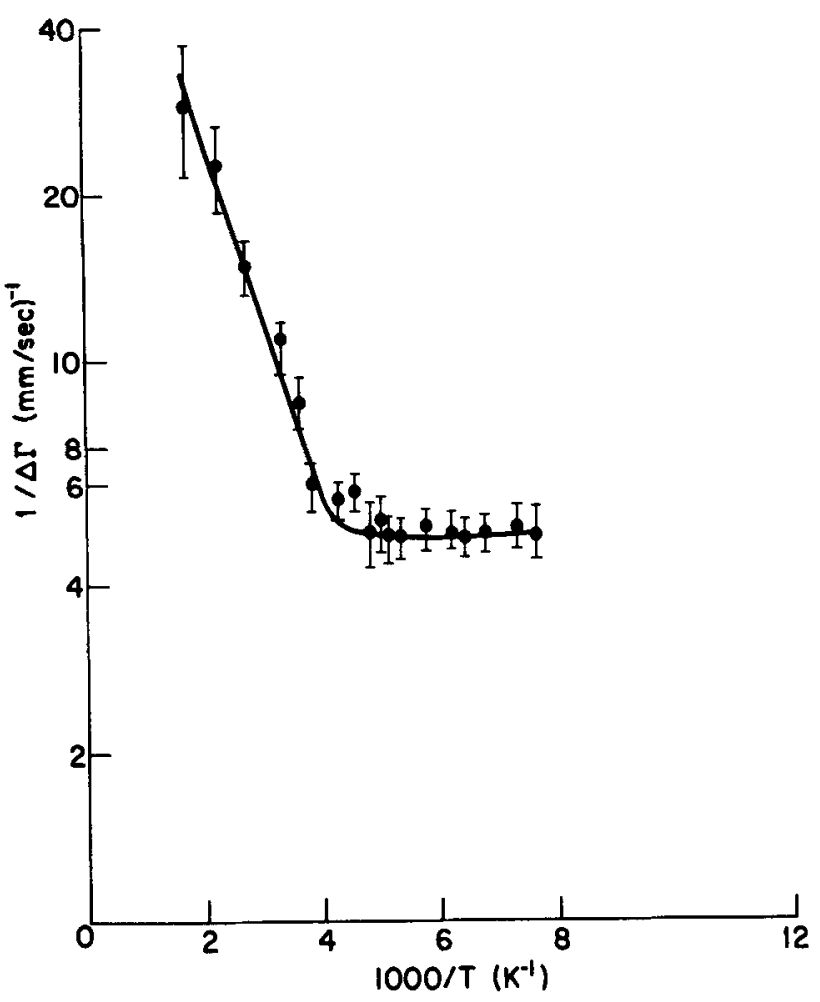

Fig. 2. Temperature dependence of the 1inewidth difference, $\Delta \Gamma$, of the $A$ and $B$ site patterns of $\mathrm{Fe}_{3} \mathrm{O}_{4}$, determined from lines $\mathrm{AA}$ and $1 \mathrm{~B}$ of the Mössbauer spectrum, as reported in ref. 19.

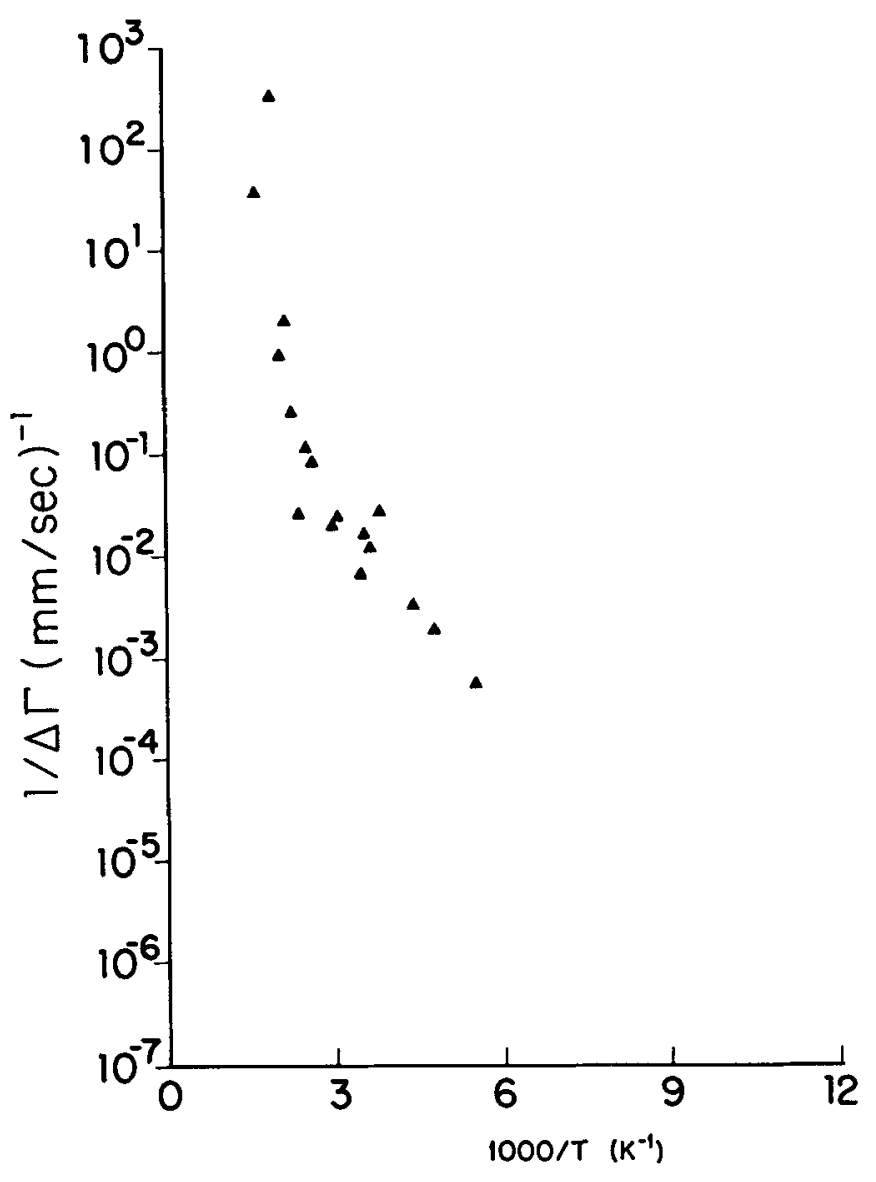

Fig. 3. Temperature dependence of the magnitude of the linewidth difference, $\Delta \Gamma$, of the $A$ and $B$ site patterns of $\mathrm{Fe}_{3} \mathrm{O}_{4}$, determined from lines $\mathrm{IA}$ and $1 \mathrm{~B}$ of the Mössbauer spectrum. This study.

was conducted only for temperatures below $300 \mathrm{~K}$. A later study ${ }^{19}$ extended these measurements over a larger temperature range; and in contrast to the earlier study ${ }^{18}$, the difference between $\Gamma_{1 A}$ and $\Gamma_{1 B}$ was found to be independent of temperature below $250 \mathrm{~K}$ (Fig. 2). However, it was just in this temperature interval that the temperature dependence of $\left(\Gamma_{B}^{2}-\Gamma_{\AA}^{2}\right)$ was used to deduce the relaxation time in the earlier study $^{18}$. Disagreement between the temperature dependence of $\left(T_{1 B}-T_{I A}\right)$ and the temperature dependence of the conductivity was also noted in this latter study. Our measurements of the temperature dependence of the linewidths of the $\mathrm{A}$ and $\mathrm{B}$ site ${ }^{57} \mathrm{Fe}$ Mössbauer patterns reveals the temperature dependence shown in Fig. 3. These results are in contrast to those reported in Reference 19 inasmuch as $\Gamma_{I B}-\Gamma_{I A}$ is found to be temperature dependent over the entire temperature range from $800 \mathrm{~K}$ to temperatures just above the Verwey transition. However, for $T>300 \mathrm{~K}$, the decrease in $\left(\Gamma_{1 \mathrm{~A}}-\right.$ $\Gamma_{I B}$ ) is not due to a decreasing $\Gamma_{B}$ as demanded by $a$ hopping model but rather to an increase in $\Gamma_{A}$ (cf. Fig. 4). In another recent study ${ }^{20}$ the decrease in $\left(\Gamma_{A}-\Gamma_{B}\right)$ with increasing temperature above $300 \mathrm{~K}$ was also found to be due more to an increase in $\Gamma_{A}$ than to a decrease in $\Gamma_{B}$. For example, at $296 \mathrm{~K} \Gamma_{1 \mathrm{~A}}=0.349$ $\mathrm{mm} / \mathrm{sec}$ and $\Gamma_{1 B}=0.392 \mathrm{~mm} / \mathrm{sec}$ and at $703 \mathrm{~K} \Gamma_{1 \mathrm{~A}}=0.376$ $\mathrm{mm} / \mathrm{sec}$ and $\Gamma_{1 B}=0.371 \mathrm{~mm} / \mathrm{sec}^{20}$. Therefore, in the temperature interval $300<T<T_{N}$, the decrease in $\left(T_{1 A}\right.$ - $\Gamma_{T_{B}}$ ) is not simply related to electron hopping among the octahedral sites; and it is reasonable to conclude that the temperature dependence of $\Gamma_{B}-\Gamma_{A}$ is not indicative of electron hopping.

Further, it has been found that $\Gamma_{B}$ is larger than $\Gamma_{A}$ even in spinels that have very low electrical conductivities 21,22 . Then too, there is rather straightforward interpretation for $\Gamma_{B}$ being larger at some temperatures than $\Gamma_{A}$. Evans ${ }^{23}$ was the first to suggest that the apparent broadening of the $B$ site lines 


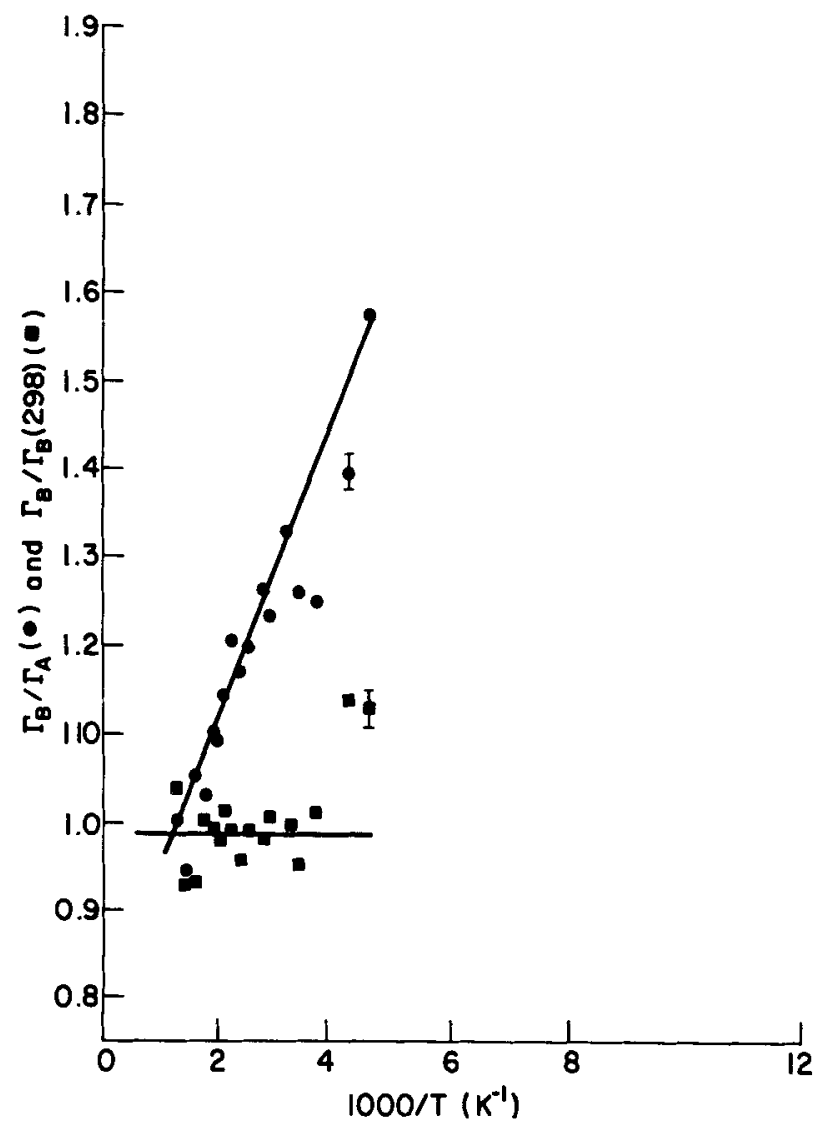

Fig. 4. Temperature dependence of $\Gamma_{1 \mathrm{~A}}(\bullet)$ and $\Gamma_{1 \mathrm{~B}}$ (•), normalized to $\Gamma_{1 \mathrm{~B}}$ at $298 \mathrm{~K}$. This study.

was not due to electron hopping but rather resulted from the different quadrupole splittings at the $B$ site as a consequence of the different angles between the principal axis of the electric field gradient tensor and the magnetic hyperfine field. That is to say, the shift of the absorption lines due to the electric quadrupole interaction is given by

$$
\varepsilon_{\mathrm{m}_{I}}=(-I)^{\left(\left|\mathrm{m}_{I}\right|+1 / 2\right)} \mathrm{e}^{2} \mathrm{qQ}\left(3 \cos ^{2} \theta-I\right) / 8
$$

where $\theta$ is the angle between the magnetic field and the principal axis of the electric field gradient tensor; and for spinel ferrites with the easy axis of magnetization being [IIl], there are two values of $\theta, \theta=$ 0 and $\theta=70^{\circ} 54^{\prime \prime}$, occurring with a relative frequency of 1 and 3, respectively. Therefore, there will be two patterns for the B site iron ions. A similar analysis would also apply for any anisotropy in the magnetic hyperfine field. Susequently, another Mössbauer investigation of $\mathrm{Fe}_{3} \mathrm{O}_{4}{ }^{24}$ has led to a similar conclusion to that of Evans $^{23}$ regarding the broadening of the $B$ site lines.

Two $B$ site patterns have also been resolved using $\mathrm{NMR}^{16,25}$ but in these instances the splitting of the $B$ site pattern is observed to rise from the anisotropy in the magnetic hyperfine field. The results of the two measurement techniques are, however, in accord; the lattice sums that lead to the anisotropy in the magnetic hyperfine field are also the ones that lead to a non-vanishing electric field gradient tensor. The different interpretations are due, on the one hand, to the much higher resolution of the NMR measurements, and on the other hand, to the fact that the electric quadrupole interaction is not detected in the NMR measurements. There is in all probability a splitting of the $B$ site pattern due to both an anisotropic magnetic hyperfine field and a non-zero electric quadrupole interaction.

As has been recognized previous $l y^{26}$ if the conduction mechanism could be described by a localized

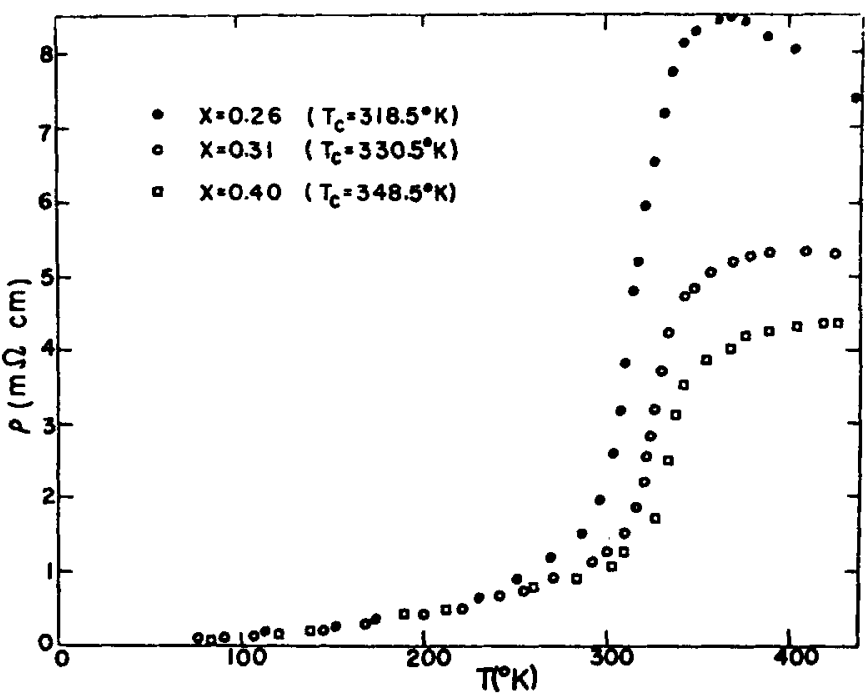

Fig. 5. Temperature dependence of the resistivity of $\mathrm{La}_{1-\mathrm{x}} \mathrm{Pb}_{\mathrm{x}} \mathrm{MnO}_{3}$, ref. 27. Note the increase in $\rho$ by almost an order of magnitude at $T_{C}$ for $x=0.26$.

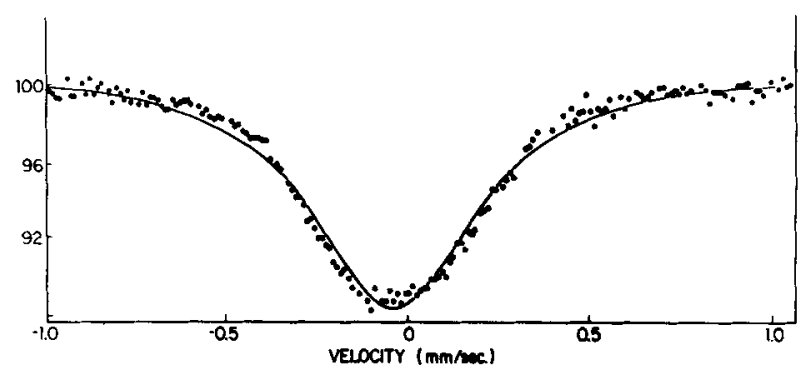

Fig. $6 .{ }^{57} \mathrm{Fe}$ Mössbauer spectrum of $\mathrm{Fe}_{3} \mathrm{O}_{4}$ above $\mathrm{T}_{\mathrm{N}}$,
ref. 28.

hopping mechanism, the magnetic double exchange mechanism would also be operative. The available data, which is rather definitive, indicate that the double exchange mechanism is not essential to the electron transport in $\mathrm{Fe}_{3} \mathrm{O}_{4}$. In materials in which the double exchange mechanism is operative, the conductivity decreases vary rapidly at the Curie temperature as shown in Fig. 5 for some $\mathrm{La}_{1-\mathrm{x}} \mathrm{Pb}_{\mathrm{x}} \mathrm{MnO}_{3}$ perovskites ${ }^{27}$. As shown in Fig. 1, this is not the case in $\mathrm{Fe}_{3} \mathrm{O}_{4}$; the decrease in the conductivity for $\mathrm{Fe}_{3} \mathrm{O}_{4}$ at $\mathrm{T}_{\mathrm{N}}$ is barely discernible. Also if the double exchange mechanism, and therefore localized hopping, was essential to the electron transport, one would expect the electron hopping relaxation time to be greater above $\mathrm{T}_{\mathbb{N}}$ than below $\mathrm{T}_{\mathrm{N}}$. In which case, the $\mathrm{Fe}^{2+}$ and $\mathrm{Fe}^{3+} \mathrm{B}$ site ions would give rise to more or less distinct hyperfine patterns. This is not the case, Fig. 6. These data which were reported earlier ${ }^{26}$ have been verified by more recent unpublished studies. The surprising aspect of these data, however, is that not only are there no distinct $\mathrm{Fe}^{2^{+}}$and $\mathrm{Fe}^{3+} \mathrm{B}$ site patterns above $\mathrm{T}_{\mathrm{N}}$ but the A site $\mathrm{Fe}^{3+}$ pattern is also unresolved.

Finally, recent resistivity, Hall effect and thermopower measurements all lead to the conclusion that the conduction mechanism in $\mathrm{Fe}_{3} \mathrm{O}_{4}$ does not involve localized hopping ${ }^{12}$. The conduction electrons are best described by band states, albeit of rather narrow widths. The apparent activation of the conductivity and the broad maximum in the conductivity can be understood in terms of a degenerate semiconductor. Theoretical studies also lead to the same conclusions ${ }^{29}$.

The neglect in the above discussion of much of the data on so-called "non-stoichiometric magnetites" has been deliberate. First of all, several investigators have demonstrated the doubtful character of the materials used in these studies ${ }^{30}$. It has also been shown 
that these "non-stoichiometric magnetites" are frequently multiphase materials and/or chemically inhomogeneous 30,31 . Microscopic measurements such as the Mössbauer effect can be understood in terms of the different phases present but macroscopic measurements such as electrical conductivity represent intractably complex convolutions of phenomena and their interactions. Many new ideas regarding conduction mechanisms in such non-stoichiometric materials resulted from these studies but it is not certain that these ideas are particularly germane to the intrinsic conductivity of $\mathrm{Fe}_{3} \mathrm{O}_{4}$. As we shall see in what follows, the technique of doping $\mathrm{Fe}_{3} \mathrm{O}_{4}$ with small quantities of other elements whose local crystal chemistries in spinel oxides are well understood and simple leads to considerably more tractable phenomena than non-stoichiometric materials.

$$
\mathrm{T} \simeq \mathrm{T}_{\mathrm{V}}
$$

Associated with the decrease in the conductivity at the Verwey transition is also a $\lambda$-type anomaly in the heat capacity ${ }^{32}$ and changes in unit cell volume and crystal symmetry ${ }^{33}, 9$. More recent measurements have, however, indicated considerably more complexity in phenomena occurring at the transition than previousiy thought. First of all, instead of one $\lambda$-type anomaly in the heat capacity versus $T$ curve, two anomalies have been observed ${ }^{11}$. Both the temperature and heat content of these anomalies depend on the amounts and kinds of impurties in $\mathrm{Fe}_{3} \mathrm{O}_{4} 34$. Secondly, the lattice of the low temperature phase is of lower symmetry than the orthorhombic or rhombohedral lattices deduced from earlier studies ${ }^{10}$. Finally, resistivity measurements for very slow heating or cooling rates in the region of the Verwey transition also exhibit two maxima ${ }^{35}$.

The main objective of the following discussion is establish the complexity of the Verwey transition and to present a possible interpretation of the systematics of the available data.

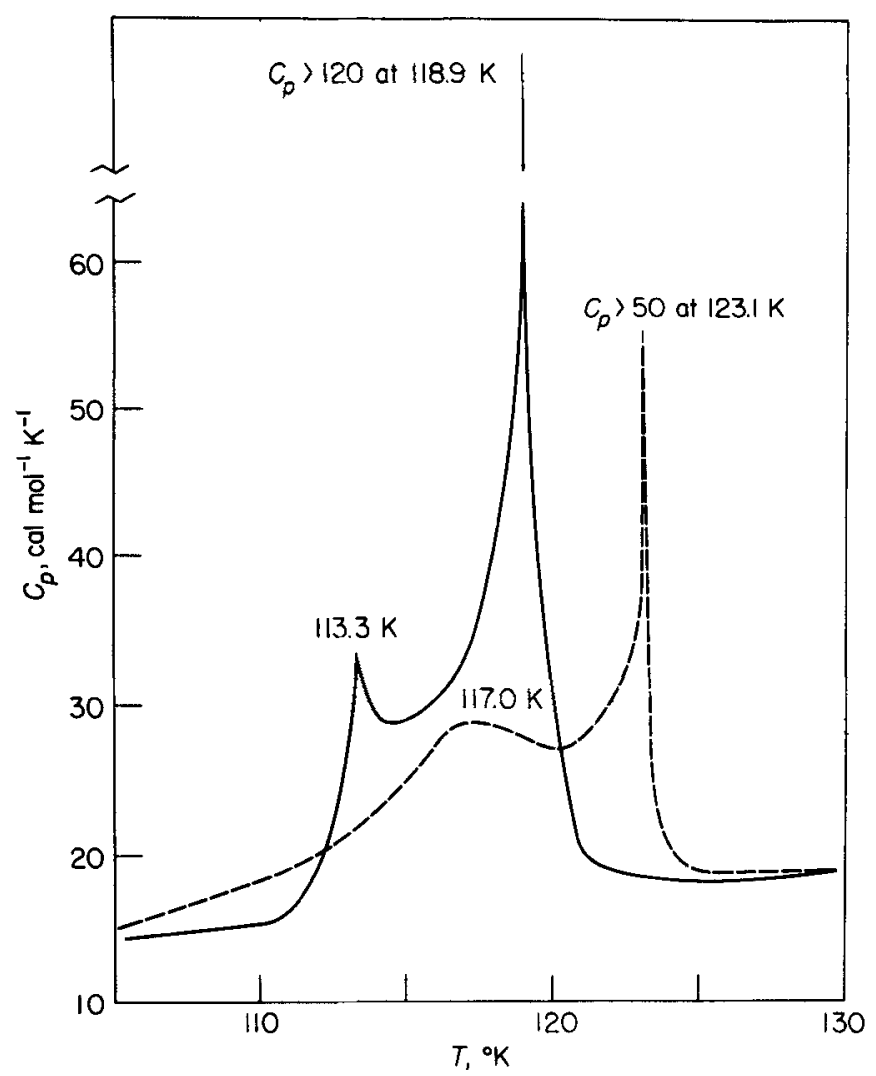

Fig. 7. Heat capacity of pure $\mathrm{Fe}_{3} \mathrm{O}_{4}(\longrightarrow)$ and

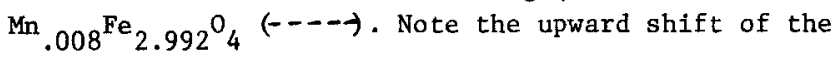
high temperature anomaly and the smearing of the lower one for $\mathrm{Mn} .008^{\mathrm{Fe}} 2.992^{\mathrm{O}} 4^{\text {. }}$
The $\mathrm{C}_{\mathrm{p}}$ versus $\mathrm{T}$ curves of $\mathrm{Fe}_{3} \mathrm{O}_{4}$ and $\mathrm{Mn}_{0.009} \mathrm{Fe}_{2.99 \mathrm{O}_{4}}$ in the vicinity of Verwey transition are shown in Fig. $7^{11}$. The bifurcated nature of the peaks and the dependence of the peak shapes on composition are noteworthy features. The enthalpies, line profiles and temperatures of the anomalies are seen to depend sensitively upon the presence of impurities: For $\mathrm{Mn}_{0.008} \mathrm{Fe}_{2.992} \mathrm{O}_{4}$ the temperature of the maximum $\mathrm{C}_{\mathrm{p}}$ in the high temperature anomaly is shifted $4 \mathrm{~K}$ from that of $\mathrm{Fe}_{3} \mathrm{O}_{4}{ }^{31}$, and the enthalpy content is $71 \mathrm{cal} / \mathrm{mol}$ compared to 98 $\mathrm{cal} / \mathrm{mol}$ for $\mathrm{Fe}_{3} \mathrm{O}_{4}{ }^{36}$. The low temperature anomaly is less sensitive to impurities; at low dopant levels its enthalpy is essentially independent of temperature but the temperature of the maximum in $\mathrm{C}_{p}$ increases ${ }^{34}$. With these new thermal data it may be concluded that (1) substitutions of closed shell ions on the A site are not innocuous; (2) the increase in temperature of the maxima in the heat capacity with decrease in $\mathrm{Fe}^{2+}$ content and/or increase in lattice constant holds for $\mathrm{Zn}^{2+}$ and $\mathrm{Cd}^{2+}$ dopant level less than 0.1 atom percent; for $\mathrm{Cd}_{0.005}$, it appears that the two anomalies occur at the same temperature; (3) at dopant levels much above 0.1 atom percent, the magnitudes of the heat capacity anomalies decrease rapidly, i.e., both anomalies are absent in a material with the composition $\mathrm{Zn}_{0.066} \mathrm{Fe}_{2} .934 \mathrm{O}_{4}$.

There is a body of literature ${ }^{37}$ that ascribed such furcation of $\lambda$-type anomalies to inhomogeneities; and this as a possible interpretation of the new thermal properties data cannot be rejected out of hand. The samples used in our studies have, however, been prepared with sufficient care to insure against inhomogeneities. Further, the furcation persists for samples prepared by a variety of different techniques and exhibits a cogent dependence on the systematics of the crystal chemistry of the dopants ${ }^{38}$.

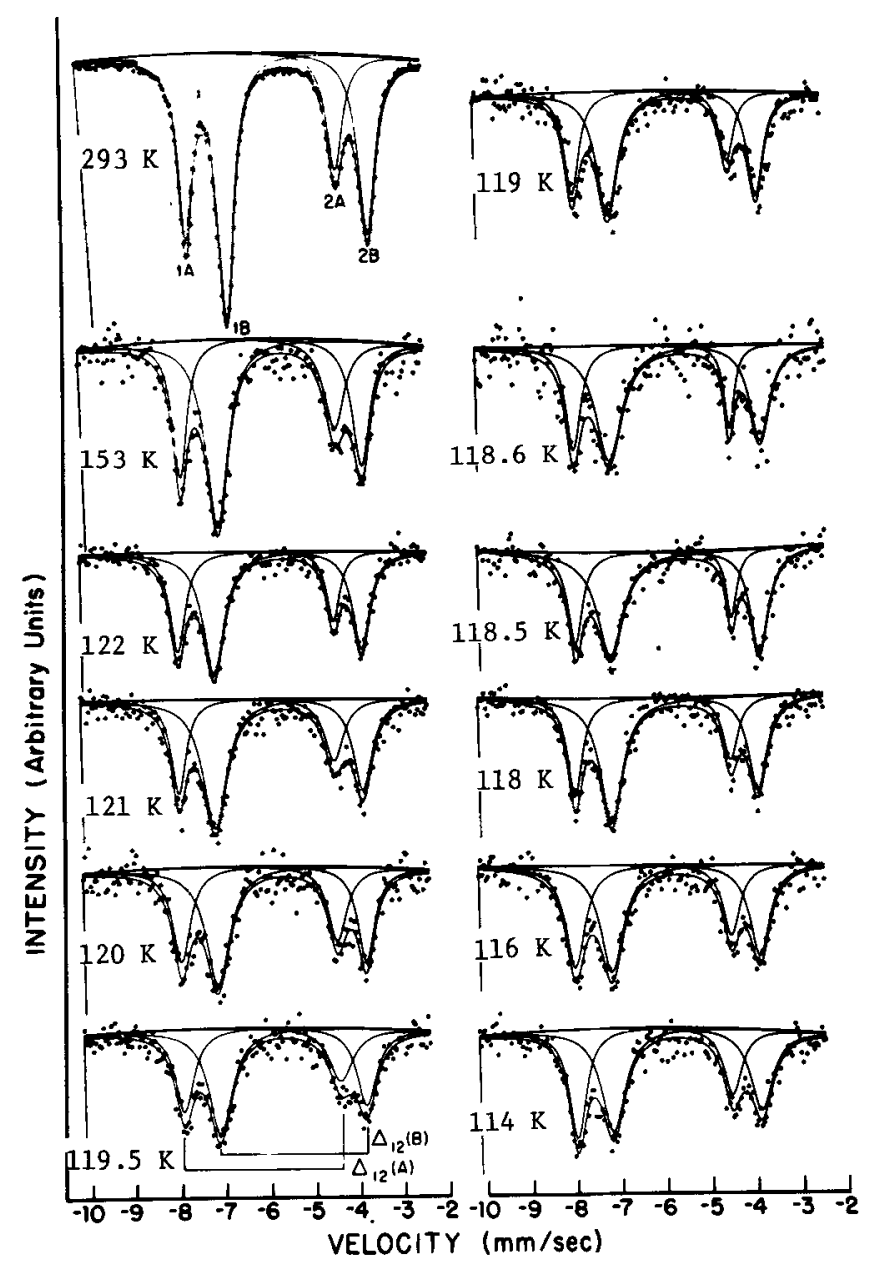

Fig. 8a. First two $A$ and $B$ site lines of the ${ }^{57} \mathrm{Fe}$ Mössbauer spectrum of $\mathrm{Zn} .005^{\mathrm{Fe}_{2}}{ }_{2.995^{\circ}}{ }_{4}$ as a function of temperature. 


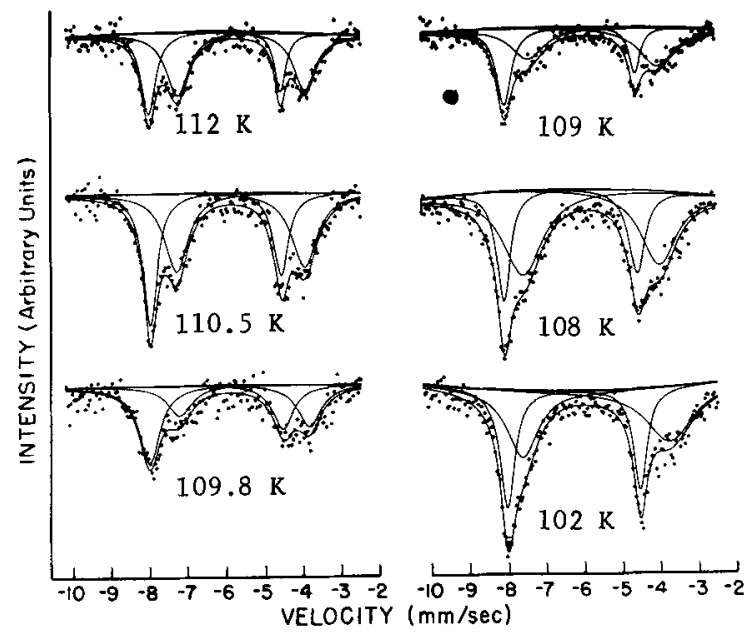

Fig. 8b. First two $A$ and $B$ site lines of the $57 \mathrm{Fe}$ Mossbauer spectrum of $\mathrm{Zn} .005^{\mathrm{Fe}_{2}} 2.995^{\circ}$ as a function of temperature.

The thermal properties measurements have proven to be crucial in delineating the complexity of the Verwey transition in $\mathrm{Fe}_{3} \mathrm{O}_{4}$, but by themselves, they provide little phenomenological insight into the crystal physics of the thermal properties variations. Therefore, ${ }^{57} \mathrm{Fe}$ Mössbauer measurements have been made in the region of the Verwey transition on the pure $\mathrm{Fe}_{3} \mathrm{O}_{4}$ and $\mathrm{Zn}_{0} 005$ $\mathrm{Fe}_{2} .99 \mathrm{O}_{4}$ samples used in the thermal properties measurements 11,35 . The most significant changes in the ${ }^{57} \mathrm{Fe}$ Mössbauer spectrum occur for the $\Delta \mathrm{m}=1,+1 / 2 \rightarrow$ $+3 / 2$ and $\Delta m=0,+1 / 2 \rightarrow+1 / 2$ transitions whose absorption lines occur in negative velocity region, Fig. 8. These four lines have been used previously to monitor the verwey transition 30 and changes in their positions and profiles have been used to confirm the complexity of the Verwey transition ${ }^{11}$. Some additional studies on non-stoichiometric magnetites have led to the conclusion that these lines are not reliable incicators of the Verwey transition ${ }^{39}$ but conclusions drawn from measurements on non-stoichiometric magnetites are to be accepted with great caution. At any rate, it is demonstrated below that a good correlation exists between the temperature of the $\lambda$-anomalies and changes in the Mössbauer spectrum for the above mentioned lines in the negative velocity region. A weak absorption line between $+2 \mathrm{~mm} / \mathrm{sec}$ and $+4 \mathrm{~mm} / \mathrm{sec}$, which is believed to be associated with more or less distinct $\mathrm{Fe}^{2^{+}}$ species ${ }^{18}$, has not been used in these measurements because of the long counting times necessary for good statistics and consequent stringent requirements on temperature stability.

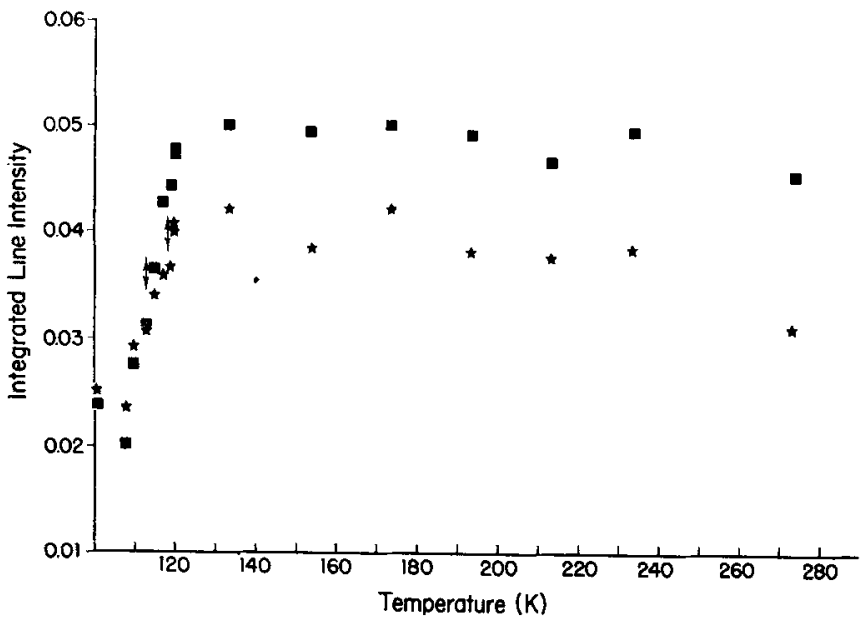

Fig. 9. Temperature dependence of the integrated intensity of lines $1(\mathbb{*})$ and $2(\boldsymbol{N})$ of the $B$ site $57_{\mathrm{Fe}}$ Mössbauer spectrum of pure $\mathrm{Fe}_{3} \mathrm{O}$. Arrows indicate the temperatures of the $\lambda$-anomalies in $C_{p}$.

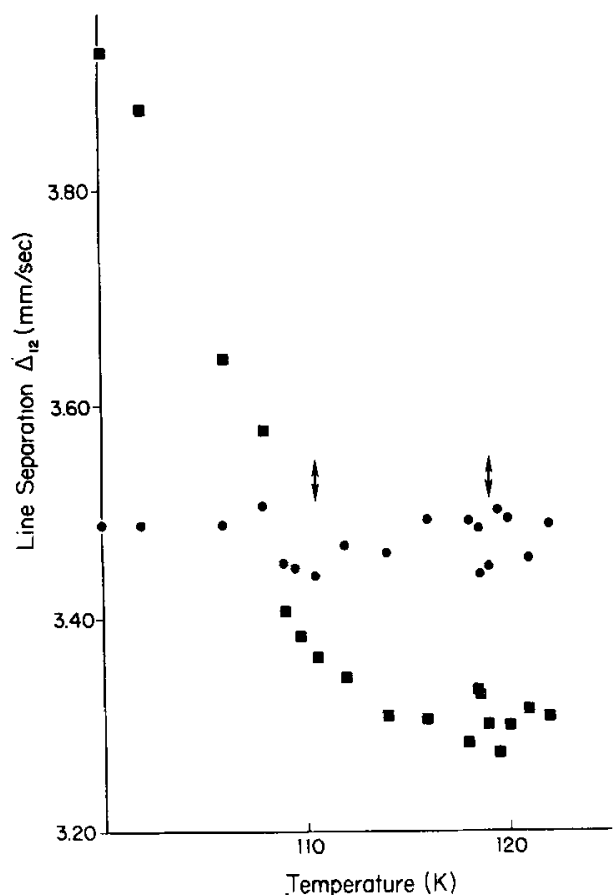

Fig. 10. Temperature dependence of the line separation, $\Delta_{12}$, (cf.Fig. 8a) of the B site ( $\boldsymbol{a}$ ) and A site (-) Mössbauer patterns of $\mathrm{Zn} .005^{\mathrm{Fe}} 2.995^{\circ}{ }_{4}$. Arrows indicate the temperatures of the $\lambda$-anomalies in $C_{p}$.

As shown in Figs. 9 and 10, the intensities and splittings $\Delta_{12}$ of the four lines show fundamental changes in the vicinity of the Verwey transition. At temperatures corresponding to the high temperature $\lambda$-anomaly the relative intensities of the lines undergo rapid change whereas the splittings, $\Delta_{12}$ while showing a greater scatter of values, has nearly the same value above and below the temperature corresponding to this first $\lambda$-anomaly. At the lower temperature heat capacity anomaly, however, $\Delta_{12}$ and the relative line intensities, both, undergo very rapid changes. There is, thus a $1: 1$ correspondence between dramatic changes in the relative line intensities, the splittings, $\Delta_{12}$, or both and the anomalies in the heat capacity. It is possible to advance a tentative explanation of these results which are consistent with and lend support to a band description of the conduction electrons above and below the Verwey transition.

If the Verwey transition is a semimetal or degenerate semiconductor $\rightarrow$ nondegenerate semiconductor transition $^{12}$, then the differences in the electronic structure above and below $\mathrm{T}_{\mathrm{V}}$ are primarily quantitative and not qualitative in nature. Therefore, this transition is to be associated with the change in the relative intensity of the lines in the Mössbauer pattern at a temperature roughly the same as that of the high temperature heat capacity anomaly. The extreme sensitivity of the temperature and line profile of this anomaly to impurities is consistent with its being associated with the degenerate-nondegenerate semiconductor transition since this transition is expected to be rather sensitive to changes in the electron concentration.

The changes in the splittings, $\Delta_{12}$, of the $B$ site pattern results either from a change in the electric quadrupole splitting, the magnetic hyperfine field, or both and are indicative of major changes in the overall structure and local site symmetries since changes in local site symmetry are expected to influence substantially the orbital angular momentum contribution to the magnetic hyperfine field and the electric quadrupole interaction. It is concluded that the low temperature heat capacity anomaly and the changes in the Mossbauer spectrum are associated with major structural changes. The relative magnitudes of the maxima observed in resistivity measurements ${ }^{32}$ are consistent with this interpretation of the heat capacity anomalies and the 
Mössbauer spectra.

\section{SUMMARY}

A reasonably consistent description of the electrical conduction mechanism and Verwey transition in $\mathrm{Fe}_{3} \mathrm{O}_{4}$ seems to be the following: Above the Verwey transition $\mathrm{Fe}_{3} \mathrm{O}_{4}$ is a semimetal or degenerate semiconductor with the conduction electrons being appropriately described by a band model. There are no data that uniquely support a localized hopping model. There is a semimetal or degenerate semiconductor $\rightarrow$ nondegenerate semiconductor transition at $\simeq 120 \mathrm{~K}$ that is accompanied by a small increase in the resistivity. This transition may be related in its origin to a Mott-Wigner metal-nonmetal transition. Previous concerns 26 about a Mott-Wigner description of the transition were based on measurements of impure magnetites that were in fact too impure to provide a test of the effect of a decrease in electron density on the temperature of the electronic transition. Impurity levels sufficiently low that they can be regarded as small perturbations on the lattice and electron concentration yield an increase in the transition temperature as expected for a Mott-Wigner transition.

At a lower temperature, there is a phase transition that is accompanied by major structural changes, as well as changes in the electrical properties. The temperature interval between these two transitions is affected by the amounts and kinds of impurities. Hence, the two transitions may occur at the same temperature for some impure samples. Below the Verwey transition the conduction electrons are in band states and distinct $\mathrm{Fe}^{2+}$ and $\mathrm{Fe}^{3+}$ oxidation states for all of the iron species do not obtain. Whether the absence of distinct $\mathrm{Fe}^{2+}$ and $\mathrm{Fe}^{3+}$ states for all of the iron atoms is due to the complexity of the structure of the low temperature phase or to charge density waves 40 is one of the questions to be resolved by further study.

Further measurements on samples with A site impurity levels slightly below and above 0.1 atom percent would be useful. The kind of dopant should also be extended to include those that occupy the octrahedral sites. A particulariy important measurement would be the determination of the resistivity at very slow heating and cooling rates in the vicinity of the Verwey transition. Lastly, further conductivity and Mössbauer measurements on pure and doped $\mathrm{Fe}_{3} \mathrm{O}_{4}$ samples above the Neel temperature are desiderata; the ${ }^{57} \mathrm{Fe}$ Mössbauer spectrum above $T_{N}$ is consistent with a band theoretical description of the conduction process but the spectrum is sufficiently perplexing and suggestive of extensive electron delocalization over both the $A$ and $B$ sites to warrant further studies.

\section{ACKNOWLEDGMENT}

Support of the work by the National Science Foundation, Grant GH-41419 is gratefully acknowledged. Additional support from the Sloan Foundation is also acknowledged.
REFERENCES

$+\quad$ Alfred P. Sloan Research Fellow.

1. D. Adler, Solid State Physics, Vo1. 2l, F. Seitz, D. Turnbull, and H. Ehrenreich, eds., (Academic Press, New York, 1968) pp. 1-113.

2. J. B. Goodenough, Progress in Solid State Chemistry, Vol. 4, H. Reiss, ed. (Pergamon, New York, 1972), pp. 145-399.

3. N. F. Mott, Phil. Mag. 20, 163 (1969).

4. E. A. Callen, Phys. Rev. 150, 367 (1966).

5. E. J. W. Verwey and P. W. Haayman, Physica $\underline{8}, 979$ (1941).

6. P. A. Miles, W. B. Westphal, and A. von Hippel, Rev. Mod. Phys. 29, 279 (1957) and references therein.

7. J. R. Drabble, T. D. Whyte, and R. M. Hooper, Solid State Commun. 2, 275 (1971).

8. Yu. D. Tropin and A. A. Lepishev, Sov. Phys.Solid State 14, 2654 (1973).

9. W. C. Hamilton, Phys. Rev. 110, 1050 (1958).

10. T. Yamada, K. Suzuki and S. Chikazumi, Appl. Phys. Letters 13,172 (1968).

11. B. J. Evans and E. F. Westrum, Jr., Phys. Rev. B5, 3791 (1972) and references therein.

12. W. J. Siemons, I.B.M. J. Res. Develop. 14, 245 (1970).

13. R. Bauminger, S. G. Cohen, S. Ofer and E. Segal, Phys. Rev. 122, 1447 (1961).

14. B. J. Evans and S. S. Hafner, J. Appl. Phys. 40, 1411 (1969).

15. R. S. Hargove and W. Kundig, Solid State Commun. 8, 303 (1970).

16. $\bar{M}$. Rubinstein, G. A. Stauss and F. Bruni, AIP Conf. Proc. 10, 1384 (1973).

17. F. Hartmann-Boutron and P. Imbert, J. Appl. Phys. 39, 775 (1968).

18. W. Kundig and R. S. Hargove, Solid State Commun. 7, 223 (1969).

19. G. A. Sawatzky, J. M. D. Coey and A. H. Morrish, J. App1. Phys. 40, 1402 (1969).

20. H. Topsde, Ph.D. Thesis, Stanford University (University Microfilms, Ann Arbor, 1974), p. 137.

21. B. J. Evans and S. S. Hafner, J. Phys. Chem. Solids 29, 1573 (1968).

22. B. J. Evans and L. J. Swartzendruber, J. Appl. Phys. 42, 1628 (1971).

23. B. J. Evans, AIP Conf. Proc. 5, 296 (1971).

24. C. I. Nistor, Rev. Roum. Phys. 18, 867 (1973).

25. N. M. Kovtun and A. A. Shamyakov, Solid State Commun. 13, 1345 (1973).

26. A. Rosencwaig, Canad. J. Phys. 47, 2309 (1969).

27. C. W. Searle and S. T. Wang, Canad. J. Phys. 48 2023 (I970).

28. B. J. Evans, AIP Conf. Proc. 10, 1398 (1972).

29. D. Ihle and B. Lorenz, Phys. Stat. Sol. 58, 79 (1973) and references therein.

30. H.-P. Weber, Ph.D. Thesis, University of Chicago, 1972, p. 62.

31. H. Topsфe, J. A. Dumesic, and M. Boudart, J. de Phys. (Paris), In Press.

32. R. W. Millar, J. Amer. Chem. Soc. 51, 215 (1929).

33. L. R. Bickford, Jr., Rev. Mod. Phys. 25, 75 (1953).

34. J. J. Bartel and E. F. Westrum, Jr., AIP Conf. Proc. 10, 1393 (1974).

35. S. Iida, M. Yamamoto, and S. Umemura, AIP Conf. Proc. 18, 913 (1974).

36. J. J. Bartel, Ph.D. Thesis, University of Michigan, 1974.

37. B. Ya. Sukarevskii, A. V. Alapina, Yu. A. Dushechkin, T. N. Kharchenko, and I. S: Shchetkin, Sov. Phys.-JETP 31, 820 (1970).

38. J. J. Bartel and E. F. Westrum, Jr., These Proceedings.

39. V. P. Romanov, V. D. Checherskii, and V. V. Eremenko, Phys. Stat. Sol. 9, 713 (1972).

40. J. B. Sokoloff, Phys. Rev. 5, 4496 (1972). 